\title{
Impact of education on ventilator-associated pneumonia in the intensive care unit
}

\author{
Pathmawathi Subramanian ${ }^{1}$, DHSci, MEd, Kee Leong $\underline{C h o y}^{2}$, MSc, MEd, Suresh Venu Gobal ${ }^{3}$, FANZCA, MMed, \\ Marzida Mansor ${ }^{3}$, MAnaes, MD, Kwan Hoong ${\underline{\mathrm{Ng}^{4}}}^{\text {, MSc, PhD }}$
}

\begin{abstract}
INTRODUCTION Ventilator-associated pneumonia (VAP) is a common risk among critically ill ventilated patients. This study aimed to investigate the effects of nurse-led education on: (a) knowledge of and compliance with ventilator care bundle (VCB) practices among intensive care unit (ICU) nurses; and (b) reduction in the rates of VAP post intervention. METHODS A quasi-experimental design with pretest-posttest evaluation and observation was used to investigate nurses' knowledge of and compliance with VCB practices, and the incidence of VAP. The study was conducted among 71 nurses, and the intervention involved structured education on VAP and its prevention using VCB in an ICU setting. Data were analysed using descriptive and inferential statistics.

RESULTS Nurse-led education significantly increased nurses' knowledge of ( $t[70]=-36.19 ; p<0.001)$ and compliance with $(t[65]=-21.41 ; p<0.001)$ VCB practices. The incidence of VAP, which was 39 per 1,000 ventilator days during the two-month period before intervention, dropped to 15 per 1,000 ventilator days during the two-month period following intervention.

CONCLUSION Our findings show that nurse-led education on VAP and VCB significantly increased knowledge of and compliance with VCB practices among ICU nurses, and was associated with a reduction in the incidence of VAP among intubated and mechanically ventilated ICU patients. Inclusion of recent knowledge and evidence-based VCB guidelines for VAP prevention when educating anaesthetists, nurses, physiotherapists and other healthcare providers in the critical care setting is recommended.
\end{abstract}

Keywords: compliance, intensive care, knowledge, ventilator-associated pneumonia, ventilator care bundle

\section{INTRODUCTION}

Ventilator-associated pneumonia (VAP) is a nosocomial airway infection of the lung parenchyma that develops more than 48-72 hours after a patient is intubated and mechanical ventilation is initiated.(1) VAP is the leading cause of death among critically ill patients, with its associated mortality rate exceeding that of other nosocomial infections such as central line catheter infection, sepsis and respiratory infections. (2) VAP has been identified as a major safety issue among critically ill patients receiving mechanical ventilation. ${ }^{(3)}$ Several studies have highlighted VAP as one of the most common healthcare-associated infections among critically ill patients on mechanical ventilation support. ${ }^{(4,5)}$ The main contributing factor of VAP is microaspiration of oropharyngeal organisms from around the endotracheal tube's cuff into the distal bronchi, which is followed by proliferation of bacteria and its invasion of lung parenchyma. ${ }^{(6)}$ Prevention of VAP is thus of utmost importance in critical care. Steps to reduce the incidence of VAP have been identified based on clinical best practice guidelines worldwide. ${ }^{(7)}$ These guidelines promulgate the ventilator care bundle (VCB) as an evidence-based guideline for the prevention of VAP. ${ }^{(4)}$ According to the Institute for Healthcare Improvement (IHI), United States of America, the VCB is a series of small, straightforward sets of practices or interventions related to ventilator care that, when implemented together, would achieve significantly better outcomes than when implemented individually. ${ }^{(7,8)}$ The VCB has four key components: (a) elevation of the head of the bed; (b) daily sedation hold; (c) gastric ulcer prophylaxis; and (d) deep vein thrombosis (DVT) prophylaxis..$^{(1,3,7-9)}$

The nurses recruited for our study belong to an intensive care unit (ICU) that did not have any structured VCB guidelines enforced prior to the study. In view of the absence of VCB guidelines, we opined that significant value can be derived from educating critical care nurses in such units. This study aimed to determine the effects of nurse-led VCB education on the incidence of VAP among ventilated patients in a critical care setting. The central query that the study set out to answer was this: what effects would the implementation of a nurse-led education programme on VCB have on the enhancement of knowledge and compliance among nurses in the ICU?

\section{METHODS}

A quasi-experimental study using a pretest-posttest design was conducted in an ICU of a large teaching hospital in Malaysia. Convenience sampling was used. All 71 nurses from the ICU were included in the study, as the general rule of thumb in the determination of sample size is to select as large a sample as possible from the target population to minimise the occurrence

${ }^{1}$ Department of Nursing Science, University of Malaya, ${ }^{2}$ College of Nursing, University Malaya Medical Centre, ${ }^{3}$ Department of Anaesthesiology, ${ }^{4}$ Department of Biomedical Imaging, University of Malaya, Kuala Lumpur, Malaysia

Correspondence: Dr Pathmawathi Subramanian, Senior Lecturer, Department of Nursing Science, University of Malaya, 50603 Kuala Lumpur, Malaysia. pathmawathi@um.edu.my 
Table I. Modified Clinical Pulmonary Infection Score adapted from Singh et al(9)

\begin{tabular}{|c|c|c|c|}
\hline \multirow[t]{2}{*}{ Clinical parameters } & \multicolumn{3}{|c|}{ Clinical Pulmonary Infection Score } \\
\hline & Grade 0 & Grade 1 & Grade 2 \\
\hline Tracheal secretions & Rare & $\begin{array}{l}\text { Abundant and nonpurulent } \\
\text { (white or light yellow in colour) }\end{array}$ & $\begin{array}{l}\text { Abundant and purulent (yellow, } \\
\text { green or brown in colour) }\end{array}$ \\
\hline Chest radiography & No infiltrate & Diffused infiltrate & Localised infiltrate \\
\hline Temperature $\left({ }^{\circ} \mathrm{C}\right)$ & $\geq 36.5$ to $\leq 38.4$ & $\geq 38.5$ to $\leq 38.9$ & $\geq 39.0$ and $\leq 36.0$ \\
\hline Arterial oxygenation $\left(\mathrm{PaO}_{2} / \mathrm{FiO}_{2}[\mathrm{mmHg}]\right)$ & $>240$ or ARDS & - & $\leq 240$ and no evidence of ARDS \\
\hline Microbiology (culture of tracheal aspirates) & Negative & - & Positive \\
\hline
\end{tabular}

ARDS: acute respiratory distress syndrome; $\mathrm{PaO}_{2} / \mathrm{FiO}_{2}$ : ratio of aveolar oxygen tension to fractional inspired oxygen concentration

of potential errors. Data collection was performed in three phases.

In Phase I, a pretest session was conducted for all 71 nurses to determine the level of their knowledge on VAP and VCB. This pretest made use of a self-constructed, 20-item tool (regarding the knowledge of VAP and its prevention via VCB practices) to test the preintervention knowledge levels of the group. Cronbach's alpha reliability coefficient for the knowledge test questions was 0.72. Two experts in critical care verified the content validity of the test questions. The pretest consisted of two parts - part A contained five questions on the demographic characteristics of the enrolled nurses, while part B contained 20 multiple-choice questions pertaining to the definition and diagnosis of VAP, its pathophysiology, risk factors, causative factors and disease burden, as well as questions on VCB. For each correct answer, one point was awarded. Nurses with total scores $>80 \%$ were considered to have good knowledge.

To determine the current practice patterns regarding ventilated patients, observation was carried out with a checklist one month before educational intervention. The observation checklist revolved around five important elements (based on the adapted VCB) to be performed: (a) head-of-bed elevation - a semirecumbent position that could be achieved by elevating the head of the bed to an angle of $30^{\circ}-45^{\circ}$; (b) daily sedation hold; (c) peptic ulcer disease prophylaxis using pantoprazole or ranitidine, as prescribed by the physician; (d) DVT prophylaxis via administration of subcutaneous heparin or enoxaparin $\left(\right.$ Clexane $\left.^{\circledR}\right)$ and application of antiembolism stockings such as thromboembolic deterrent stockings as prescribed; and (e) daily oral care with the help of a suction toothbrush and chlorhexidine gluconate $0.05 \%$.

The rationale behind using these VCB bundles was based on literature review, local guidelines and discussions with ICU specialists at the study unit. It was the nurses' responsibility to implement all the elements identified above. A scoring system where one mark was awarded for implementation of each of the above five VCB elements was applied to determine compliance with VCB practices. Compliance was determined by a score of $5 / 5$, as the all-or-none principle was selected as the measurement criterion for determining compliance and noncompliance. Each nurse was observed twice, at any time, and the average of the two scores was recorded. The role of complete observer was adopted in this study. The nurses were observed during shift reports at 0700 hours and 1400 hours, and throughout the morning and afternoon shifts.

The Clinical Pulmonary Infection Score (CPIS), which has previously been used to determine the development of $\operatorname{VAP}^{(9,10)}$ was used in our study. Using the CPIS checklist, incidences of VAP were observed over a two-month period before the implementation of educational intervention on VCB for the ICU nurses. The decision to use CPIS followed our review of several studies ${ }^{(9,10)}$ in the literature as well as discussion with the consultant anaesthetist and intensivist at the study unit. Data on new occurrence of VAP were collected for new ICU admissions for two months. Our study made use of a modified CPIS consisting of six clinical and laboratory variables: (a) tracheal secretions; (b) chest radiography; (c) body temperature; (d) leucocyte count; (e) arterial oxygenation (ratio of alveolar oxygen tension $\left[\mathrm{PaO}_{2}\right]$ to fractional inspired oxygen concentration $\left[\mathrm{FiO}_{2}\right]$ ); and (f) microbiology (culture of tracheal aspirates). ${ }^{(9,10)}$ The CPIS varied from $0-12,{ }^{(9,10)}$ with a CPIS score $>6$ being an excellent predictive value of high burden of bacteria in the lower respiratory tract in the diagnosis of VAP. ${ }^{(10)}$ Using the checklist, the modified baseline scores were assessed and calculated based on the first five variables of the CPIS (Table I). Gram staining and culture scores were incorporated into the CPIS baseline score by adding two points when Gram staining or culture was positive. Therefore, a CPIS score $>6$ at baseline, or after incorporating Gram staining or culture results, was suggestive of pneumonia when accompanied by the presence of a new lung infiltrate on chest radiographs after admission, with at least two of the following clinical signs and symptoms: (a) purulent tracheal secretions; (b) body temperature $>39^{\circ} \mathrm{C}$; (c) leucocytosis $\left(>11,000\right.$ cells $\left./ \mathrm{mm}^{3}\right)$ or leucopenia $\left(<4,000\right.$ cells $\left./ \mathrm{mm}^{3}\right)$; (d) ratio of $\mathrm{PaO}_{2}$ to $\mathrm{FiO}_{2}$ $\leq 240$ with no evidence of acute respiratory distress syndrome; and (e) positive culture of tracheal aspirates. This study excluded patients with a clinical diagnosis of pneumonia at the time of intubation (confirmed by chest radiography) and those with head injuries, as sedation hold is contraindicated in such patients.

Phase II comprised structured educational intervention, which was provided after the completion of pretest evaluations. The purpose of the educational programme was to provide 
Table II. Demographic characteristics of nurses enrolled in the study $(n=71)$.

\begin{tabular}{lr}
\hline Characteristic & No. (\%) \\
\hline Gender & \\
$\quad$ Male & $4(5.6)$ \\
Female & $67(94.4)$ \\
Age $^{*}$ (yrs) & $26.10 \pm 4.99$ \\
Range (yrs) & \\
$21-31$ & $63(88.7)$ \\
$32-41$ & $8(11.3)$ \\
Education level & \\
Diploma & $63(88.7)$ \\
Post-basic diploma & $8(11.3)$ \\
Work experience in critical care (yrs) & \\
$<1$ & $15(21.1)$ \\
$1-5$ & $34(47.9)$ \\
$6-10$ & $14(19.7)$ \\
$>10$ & $8(11.3)$ \\
\hline${ }^{*}$ Data is presented as mean \pm standard deviation.
\end{tabular}

Table III. Comparison of nurses' pre- and posttest knowledge $(n=71)$ and compliance $(n=66)$.

\begin{tabular}{|c|c|c|c|c|}
\hline \multirow[t]{2}{*}{ Variable } & \multicolumn{4}{|c|}{ Pre- and posttest scores } \\
\hline & Mean \pm SD & $95 \% \mathrm{Cl}$ & $t(d f)$ & p-value* \\
\hline Knowledge & $-3.28 \pm 7.64$ & -34.63 to -31.01 & $-36.19(70)$ & $<0.001$ \\
\hline Compliance & $-3.61 \pm 13.69$ & -39.43 to -32.69 & $-21.41(65)$ & $<0.001$ \\
\hline
\end{tabular}

information on and strategies for the prevention of VAP. The intervention consisted of two parts. Part I included the following information: (a) what is VAP; (b) who is susceptible to VAP; (c) pathophysiology of VAP; (d) risk factors and causes of VAP; (e) disease burden and impact of VAP; and ( $f$ ) diagnosis of VAP. Part II dealt with VAP prevention strategies and included the following adapted VCB guidelines: (a) elevation of the head of the bed to $30^{\circ}-45^{\circ}$, unless medically contraindicated; (b) daily sedation hold and assessment of readiness for extubation; (c) peptic ulcer disease prophylaxis; (d) DVT prophylaxis; and (e) daily oral care using chlorhexidine gluconate $0.05 \%$. Teaching sessions, held in the afternoon, were planned according to the nurses' working roster and assignments - nurses on morning shift attended the teaching session held from 1430 hours to 1530 hours, whereas nurses on afternoon shift attended the session held from 1300 hours to 1400 hours. These 30-minutes teaching sessions, which were mainly didactic, were conducted with the use of PowerPoint presentations in a conference room situated in the ICU. Finally, phase III consisted of posttest evaluations that were carried out among all the nurses one month after the educational intervention. In addition to these evaluations, postintervention observation was conducted over a period of two months for all the nurses. The incidence of VAP during this two-month period was determined using the modified CPIS checklist.

Ethical approval for the study was obtained from the hospital's medical ethics committee. Written consent was obtained from all participants before the nurse-led educational intervention was implemented. However, no informed consent was requested from patients' next of kin, as the collection of data regarding VAP among patients admitted to ICU during the study period was entirely observational. All statistical analyses were performed using the Statistical Package for the Social Sciences for Windows version 16 (SPSS Inc, Chicago, IL, USA). Descriptive and inferential statistics were also used. Paired sample $t$-test was used to compare the mean scores of pre- and posttest evaluations on knowledge and compliance. A p-value $<0.05$ was considered statistically significant. Comparison between pre- and postintervention incidences of VAP was performed descriptively.

\section{RESULTS}

The study achieved $100 \%$ response rates for pre- and posttest evaluations, educational intervention and preintervention observation of VCB practices among the nurses. However, the response rate for observation of VCB practices post intervention was only $93 \%$. All 71 nurses from the ICU participated in the study. The mean age of the participants was 26.10 (range 21-41) years. The demographic characteristics of the nurses who participated in the study are shown in Table II.

Results of the paired sample $t$-test revealed that the nurse-led educational intervention had a significant effect on the nurses' knowledge of VAP and VCB, as reflected in their test scores (preintervention $63.17 \pm 9.34$; postintervention $95.99 \pm 4.68$; $\mathrm{t}[70]=-36.19$; two-tailed $\mathrm{p}<0.001)$. The mean increase in knowledge test scores was -3.28 (95\% confidence interval [CI] -34.63 to -31.01$)$. Paired sample $t$-test also indicated a significant effect on the compliance scores of the nurses following nurse-led educational intervention (preintervention $60.00 \pm 11.09 ;$ postintervention $96.06 \pm 8.75 ; \mathrm{t}[65]=-21.41 ;$ two-tailed $\mathrm{p}<0.001)$. The mean increase in compliance scores was -3.61 (95\% Cl -39.43 to -32.69$)$. Table III tabulates the results of the comparison of pre- and posttest knowledge and compliance.

Table IV presents the incidence of VAP among intubated and mechanically ventilated patients in the pre- and postintervention periods. A total of 136 patients were admitted to the ICU during the two-month preintervention period, while 130 were admitted during the two-month postintervention period. However, more patients were intubated and ventilated in the postintervention period $(n=110)$ than the preintervention period $(n=101)$. Among the 101 patients who were intubated and ventilated during the preintervention period, 22 were diagnosed with VAP. Conversely, only seven out of the 110 patients in the postintervention period were diagnosed with VAP, indicating a reduction in the incidence of VAP following intervention.

\section{DISCUSSION}

Our findings indicate that education on VAP and VCB significantly increased ICU nurses' knowledge of VAP and compliance with 
Table IV. Incidence of ventilator-associated pneumonia (VAP) among patients admitted to the intensive care unit (ICU) during the study period.

\begin{tabular}{lll}
\hline Variable & \multicolumn{2}{c}{ No. (\%) } \\
\cline { 2 - 3 } & Preintervention & Postintervention \\
\hline Patients admitted to ICU & 136 & 130 \\
$\begin{array}{l}\text { Intubated and ventilated } \\
\text { patients }\end{array}$ & $101(74.26)$ & $110(84.61)$ \\
Episodes of VAP & 22 & 7 \\
Total ventilation days & 564 & 463 \\
$\begin{array}{l}\text { Incidence of VAP } \\
\text { (per 1,000 ventilator days) }\end{array}$ & 39.01 & 15.11 \\
\hline
\end{tabular}

*VAP incidence $($ per 1,000 ventilator days $)=($ episodes of VAP/total ventilation days) $\times 1,000$.

VCB practices, thereby reducing the postintervention incidence of VAP among intubated and mechanically ventilated patients. This is similar to the findings in other studies. ${ }^{(3,11)}$ Al-Tawfiq and $\mathrm{Abed}^{(4)}$ found that the rate of adherence to all VCB elements increased following intervention (preintervention $20 \%$; postintervention $82 \%$ ). Similarly, Hawe et $\mathrm{al}^{(12)}$ reported a significant decrease in incidence of VAP in an ICU, from 20.6 episodes per 1,000 ventilator days prior to intervention to 8.5 episodes per 1,000 ventilator days after intervention, indicating a $59 \%$ reduction in the incidence of VAP following intervention. Our study found that ICU nurses' compliance with VCB practices also significantly increased following intervention $(\mathrm{t}[65]=-21.41$; $\mathrm{p}<0.001)$, with a majority of nurses $(81.8 \%)$ complying with VCB practices after educational intervention, achieving 100\% compliance in the following elements: implementation of headof-bed elevation; peptic ulcer disease prophylaxis; and DVT prophylaxis (data not presented). Our results are in agreement with Tolentino-DelosReyes et al's findings of a significant increase in ICU nurses' compliance with VAP prevention strategies (such as head elevation, oral care and handwashing) following a 30-minute educational session $(p<0.001) .{ }^{(3)}$ Likewise, other studies have also reported increased adherence to all VCB elements after intervention. ${ }^{(4,13)}$ We also found that the incidence of VAP reduced by 15 episodes in the postintervention period, which is similar to that reported in other studies. ${ }^{(3,12)}$ Therefore, it is possible that this decrease in the incidence of VAP was due to educational intervention.

Our study was, however, not without its limitations. First, the study's sample size was small. Second, confounding factors due to complexity of the patients' conditions may have affected the determination of VAP. Third, the recommended strength of chlorhexidine gluconate $(0.05 \%)$ used in our study was lower than the strength of $0.12 \%$ recommended by the Boston-based $\mathrm{IHI}$, as only the use of $0.05 \%$ chlorhexidine gluconate is allowed at our study site. Longitudinal studies with a larger population will be needed to confirm the findings of our study.

In conclusion, we found that nurse-led educational intervention on VAP and VCB successfully enhanced ICU nurses' knowledge of and compliance with VCB guidelines, effecting a reduction in the postintervention incidence of VAP. Therefore, it is crucial that nurses be educated on good clinical practices in order to improve nursing care standards. Nursing services should emphasise the importance of nurse-led education based on evidence-based practice, and implement such interventions to improve nursing care. Our study provides preliminary evidence that supports the implementation of clinical best practices such as VCB guidelines in an ICU setting. It is recommended that the most recent knowledge and evidence-based VCB guidelines for VAP prevention be included in the focus of continuing medical education of doctors, nurses, physiotherapists and other healthcare personnel in the critical care setting, with the aim of improving care and reducing the incidence of VAP among intubated and mechanically ventilated ICU patients.

\section{ACKNOWLEDGEMENTS}

This study was supported by a research grant (no. PS208/2010B, RG396-2011) from the Health and Translational Cluster, Faculty of Medicine, University of Malaya, Malaysia. The authors are also grateful to all participants for their time and support.

\section{REFERENCES}

1. Westwell S. Implementing a ventilator care bundle in an adult intensive care unit. Nurs Crit Care 2008; 13:203-7.

2. Wip C, Napolitano L. Bundles to prevent ventilator-associated pneumonia: how valuable are they? Curr Opin Infect Dis 2009; 22:159-66.

3. Tolentino-DelosReyes AF, Ruppert SD, Shiao SY. Evidence-based practice: use of the ventilator bundle to prevent ventilator-associated pneumonia. Am J Crit Care 2007; 16:20-7.

4. Al-Tawfiq JA, Abed MS. Decreasing ventilator-associated pneumonia in adult intensive care units using the Institute for Healthcare Improvement bundle. Am J Infect Control 2010; 38:552-6.

5. Hunter J, Annadurai S, Rothwell M. Diagnosis, management and prevention of ventilator-associated pneumonia in the UK. Eur J Anaesthesiol 2007; 24:971-7.

6. Efrati S, Deutsch I, Antonelli M, et al. Ventilator-associated pneumonia: current status and future recommendations. J Clin Monit Comput 2010; 24:161-8

7. Institute for Healthcare Improvement. Implement the IHI Ventilator Bundle [online]. Available at: http://www.ihi.org/knowledge/pages/ changes/implementtheventilatorbundle.aspx. Accessed September 9, 2010.

8. Institute for Healthcare Improvement. Getting started kit: Preventing ventilator-associated pneumonia (100,000 lives campaign) - How-to guide.

9. Singh N, Rogers P, Atwood CW, Wagener MM, Yu VL. Short-course empiric antibiotic therapy for patient with pulmonary infiltrates in the intensive care unit. Am J Respir Crit Care Med [serial online] 2000; 162:505-11. Available at: http://www.atsjournals.org/. Accessed September 18, 2010.

10. Pugin J. Clinical signs and scores for the diagnosis of ventilator-associated pneumonia. Minerva Anestesiol [serial online] 2002; 68:261-5. Available at: http://www.minervamedica.it/. Accessed September 16, 2010.

11. Apisarnthanarak A, Pinitchai $U$, Thongphubeth $K$, et al. Effectiveness of an educational program to reduce ventilator-associated pneumonia in a tertiary care center in Thailand: a 4-Year Study. Clin Infect Dis 2007; 45:704-11.

12. Hawe CS, Ellis KS, Cairns CJ, Longmate A. Reduction of ventilatorassociated pneumonia: active versus passive guideline implementation. Intensive Care Med 2009; 35:1180-6.

13. Crunden E, Boyce C, Woodman H, Bray B. An evaluation of the impact of the ventilator care bundle. Nurs Crit Care 2005; 10:242-6. 